\title{
Systolic groups acting on complexes with no flats are word-hyperbolic
}

\author{
by \\ Piotr Przytycki (Warszawa)
}

\begin{abstract}
We prove that if a group acts properly and cocompactly on a systolic complex, in whose 1-skeleton there is no isometrically embedded copy of the 1-skeleton of an equilaterally triangulated Euclidean plane, then the group is word-hyperbolic. This was conjectured by D. T. Wise.
\end{abstract}

1. Introduction. Systolic complexes were introduced by T. Januszkiewicz and J. Świątkowski in [5] and independently by F. Haglund in [3]. These are simply connected simplicial complexes satisfying certain link conditions. Some of their properties are very similar to the properties of CAT(0) metric spaces, therefore they are also called complexes of simplicial nonpositive curvature. In particular it was shown in [5, Chapter 5] that they are contractible.

Directed geodesics are well defined for systolic complexes and one also has the notion of convexity. This was used by the authors of [5] to prove that if a group $\Gamma$ acts properly and cocompactly by simplicial automorphisms on a systolic complex, then $\Gamma$ is biautomatic, so also semihyperbolic. It was shown that if one imposes a slightly stronger condition on links (7systolicity), the complex must be a hyperbolic metric space in the sense of Gromov (for the definition see [1, Chapter III.H]). A systolic complex does not have to be hyperbolic in general, for example equilaterally triangulated Euclidean plane is a two-dimensional systolic complex. We prove that this is the only obstruction. Our result is similar in spirit to the following well known theorem.

TheOREm 1.1 ([1, Chapter III. $]])$. If a group $\Gamma$ acts properly and cocompactly by isometries on a locally compact $\mathrm{CAT}(0)$ space $X$, then $\Gamma$ is

2000 Mathematics Subject Classification: 20F67, 20F65.

Key words and phrases: systolic group, simplicial nonpositive curvature, flat plane theorem, word-hyperbolic group. 
word-hyperbolic if and only if $X$ does not contain an isometrically embedded copy of the Euclidean plane.

Not every systolic complex is a CAT(0) space and our goal is to prove a systolic analogue to Theorem 1.1.

THEOREM 1.2. Let $\Gamma$ be a systolic group acting on a systolic complex $X$. Then $\Gamma$ is word-hyperbolic if and only if there is no isometric embedding of the 1-skeleton of an equilaterally triangulated Euclidean plane into the 1-skeleton $X^{(1)}$ of $X$.

An alternative version of proof could be obtained by using a theorem of D. T. Wise [7] on minimal area embedded flat plane and the recent study by T. Elsner [2] on minimal flat surfaces in systolic complexes. Our proof, however, is more direct.

I would like to thank Jacek Świątkowski for posing the problem and advice.

2. Some information on systolic complexes. Let us recall the definition of a systolic complex and a systolic group following [5].

Definition 2.1. A subcomplex $K$ of a simplicial complex $X$ is called full in $X$ if any simplex of $X$ spanned by vertices of $K$ is a simplex of $K$. A simplicial complex $X$ is called flag if any set of vertices which are pairwise connected by edges of $X$ spans a simplex in $X$. A flag simplicial complex $X$ is called $k$-large, $k \geq 4$, if there are no embedded cycles of length $<k$ which are full subcomplexes of $X$.

Definition 2.2. A simplicial complex $X$ is called systolic if it is connected, simply connected and the links of all simplices in $X$ are 6-large. A group $\Gamma$ is called systolic if it acts cocompactly and properly by simplicial automorphisms on a systolic complex $X$. (Properly means $X$ is locally finite and for each compact subcomplex $K \subset X$ the set of $\gamma \in \Gamma$ such that $\gamma(K) \cap K \neq \emptyset$ is finite.)

Recall [5, Chapter 2] that systolic complexes are themselves 6-large. In particular they are flag. Now we will briefly treat the definitions and facts concerning convexity:

Definition 2.3. For every pair of vertices $A, B$ in a simplicial complex $X$ denote by $|A B|$ the combinatorial distance between $A, B$ in $X^{(1)}$, the 1 skeleton of $X$. A subcomplex $K$ of a simplicial complex $X$ is called 3-convex if it is a full subcomplex of $X$ and for every pair of edges $A B, B C$ such that $A, C \in K$ and $|A C|=2$, we have $B \in K$. A subcomplex $K$ of a systolic complex $X$ is called convex if it is connected and the links of all simplices in $K$ are 3 -convex subcomplexes of the links of those simplices in $X$. 
In Chapter 8 of [5] the authors conclude that convex subcomplexes of a systolic complex $X$ are contractible, full and 3-convex in $X$. Define the combinatorial ball $B_{n}(Y)=\operatorname{span}\{P \in X:|P S| \leq n$ for some vertex $S \in Y\}$, where $n \geq 0, Y \subset X$. If $Y$ is convex (in particular, if $Y$ is a simplex) then $B_{n}(Y)$ is a convex subcomplex of a systolic complex $X$, as proved in $[5$, Chapter 8].

We will need a crucial projection lemma ([5, Lemma 14]), which we will apply in most cases to $\sigma$ being edges. Define the residue of a simplex $\sigma$ in $X$ as the union of all simplices in $X$ which contain $\sigma$.

Lemma 2.4. Let $Y$ be a convex subcomplex of a systolic complex $X$ and let $\sigma$ be a simplex in $B_{1}(Y) \backslash Y$. Then the intersection of the residue of $\sigma$ and of the complex $Y$ is a simplex (in particular, it is nonempty).

Definition 2.5. The simplex arising as in Lemma 2.4 is called the projection of $\sigma$ onto $Y$.

Now for a pair of vertices $V, W$ with $|V W|=n$ in a systolic complex $X$ we define inductively a series of simplices $\sigma_{0}=V, \sigma_{1}, \ldots, \sigma_{n}=W$ as follows. Take $\sigma_{i+1}$ equal to the projection of $\sigma_{i}$ onto $B_{n-1-i}(W)$ for $i=0,1, \ldots, n-1$. The sequence $\left(\sigma_{n}\right)$ is called the directed geodesic from $V$ to $W$. Let $\gamma$ be any 1-skeleton geodesic connecting $V$ to $W$, whose consecutive vertices are contained in consecutive simplices of the directed geodesic from $V$ to $W$. In this setting we restate Proposition 7 of [5, Chapter 11].

Proposition 2.6. If $V, W$ belong to a common convex subcomplex $K$ of $X$, then $\gamma$ is also contained in $K$.

Definition 2.7. We will call any 1-skeleton geodesic $\gamma$ as in Proposition 2.6 a special geodesic $\left({ }^{1}\right)$.

3. Embedding lemmas. In this section we prepare the proof of the main theorem.

Definition 3.1. A two-dimensional simplicial complex with distinguished vertices $A, B$ and $C$ is called a $k$-triangle $A B C, k \geq 0$, if it is simplicially equivalent to equilateral triangulation into $k^{2}$ simplices of a Euclidean triangle of edge length $k$, with vertices $A, B, C$ corresponding to the vertices of the original Euclidean triangle.

Lemma 3.2. Let $D: \Delta \rightarrow X$ be a simplicial mapping from $\Delta$, a $k$ triangle $A B C$, into a systolic complex $X$ such that for any vertex $V \in$ $\{A, B, C\}$ and any vertex $P$ lying in $\Delta$ on the unique geodesic connecting the other two vertices from the set $\{A, B, C\}$ we have $|D(V) D(P)|=k$.

$\left({ }^{1}\right)$ F. Haglund and J. Świątkowski have proved in [4] that every 1-skeleton geodesic in a systolic complex is special in this sense. 
Then $D$ considered as a mapping between the 1-skeletons of $\Delta$ and $X$ is an isometric embedding.

Proof. Take any two distinct vertices $R, S$ in $\Delta$. We claim that $R, S$ lie on a certain 1-skeleton geodesic in $\Delta$ connecting a vertex $V \in\{A, B, C\}$ to some point $P$ defined as in the hypothesis of the lemma. This can be observed in the following way. Recall that the $k$-triangle $\Delta$ carries the Euclidean structure. Consider three straight Euclidean lines going through $R$ contained in the 1 -skeleton of $\Delta$. They divide $\Delta$ into six regions. Now, depending on which region vertex $S$ is in, it is easy to find vertices $V, P$ and a geodesic $V P$ containing $R$ and $S$. ( $V, P$ belong to the sector $S$ is in and to the opposite sector.) By the hypothesis of the lemma $D$ must embed the geodesic $V P$ into $X^{(1)}$, so it also preserves the 1 -skeleton distance between $R$ and $S$. This means that $D$ considered as a mapping between the 1-skeletons of $\Delta$ and $X$ is an isometric embedding.

LemmA 3.3. Let $D: \Delta \rightarrow X$ be a simplicial mapping from $\Delta, a k$ triangle $A B C$, into a systolic complex $X$ such that $|D(A) D(B)|=$ $|D(B) D(C)|=|D(C) D(A)|=k$. Denote by $A B$ the unique length $n$ path in $\Delta$ between vertices $A, B$ consisting of $k$ edges and $k+1$ vertices. If there exists a convex $Z \subset X$ such that $D^{-1}\left(B_{l}(Z)\right)=B_{l}(A B)$ for $l=0,1, \ldots, k$ then $D$ considered as a mapping between the 1-skeletons of $\Delta$ and $X$ is an isometric embedding.

Proof. Note that the hypothesis immediately implies that the distance between $D(C)$ and $D(A B)$ is equal to $k$. In order to apply Lemma 3.2 we have to prove the same for $D(B), D(A C)$ and $D(A), D(B C)$. We focus on the last pair.

Denote by $P_{i}^{j}$ the unique vertex of $\Delta$ which lies at distance $i$ in the 1-skeleton of $\Delta$ from $C$ and at distance $j$ in the 1-skeleton of $\Delta$ from $A$, $0 \leq i, j \leq k, i+j \geq k$.

We will prove by backward induction that

$$
\left|D\left(P_{i}^{k}\right) D(A)\right|=\left|P_{i}^{k} A\right|=k \quad \text { for } i=k, k-1, \ldots, 0 .
$$

For $i=k$ we have $P_{k}^{k}=B$, so $\left|D\left(P_{k}^{k}\right) D(A)\right|=|D(B) D(A)|=k=|B A|$ is already an assumption of the lemma.

Suppose we have already proved the equality for all $i$ with $0 \leq s<i \leq k$. We now prove it for $i=s$. Let $D(A)=S_{0}, S_{1}, \ldots, S_{m-1}, S_{m}=D\left(P_{s}^{k}\right)$ be the consecutive vertices of a 1-skeleton special geodesic of length $m$ joining $D(A)$ to $D\left(P_{s}^{k}\right)$ in $X$. Notice that $S_{m}$ is at distance $k-s$ from $Z$, but $S_{0}$ belongs to $Z$. Assume $r<m$ is greatest such that $S_{r} \in B_{k-s-1}(Z)$. Due to convexity of balls the vertices $S_{q}$ with $m \geq q>r$ belong to $B_{k-s}(Z)$. Now for each edge $S_{q} S_{q+1}$ with $r<q<m$ choose a point $R_{q}$ in $B_{k-1-s}(Z)$ contained in the projection of $S_{q} S_{q+1}$ onto $B_{k-1-s}(Z)$. By 
the projection properties (Lemma 2.4) the sequence of vertices $D(A)=$ $S_{0}, S_{1}, \ldots, S_{r}, R_{r+1}, R_{r+2}, \ldots, R_{m-1}, D\left(P_{s+1}^{k}\right)$ is connected by edges in the 1-skeleton of $X$ and therefore by induction hypothesis we have $m \geq k$. By choosing a path in $X$ between $D(A)$ and $D\left(P_{s}^{k}\right)$ which is an image of a geodesic path between $A$ and $P_{s}^{k}$ in $\Delta$ one sees that $\left|D(A) D\left(P_{s}^{k}\right)\right| \leq k$, so altogether $\left|D(A) D\left(P_{s}^{k}\right)\right|=k$, as desired.

In this way we have proved that the distance between $D(A)$ and $D(B C)$ is $k$. By repeating the same argument we also find that $\left|D(B) D\left(P_{i}^{j}\right)\right|=k$ for any $i, j \geq 0$ with $i+j=k$. Now we know that the distances in $X^{(1)}$ between $D(A), D(B), D(C)$ and the vertices which are images of the opposite edges in the $k$-triangle $\Delta$ are all equal to $k$, so we can apply Lemma 3.2.

LEMMA 3.4. Let $\Gamma$ be a group acting cocompactly on a locally finite systolic complex $X$. If for arbitrarily large $n>0$ there exists an isometric embedding of the 1-skeleton of an n-triangle $\Delta$ into $X^{(1)}$, then there exists an isometric embedding of the 1-skeleton of an equilaterally triangulated Euclidean plane into $X^{(1)}$.

Proof. Denote by $E$ an equilaterally triangulated Euclidean plane and by $\Delta_{0}$ any vertex of $E$. For all $k \geq 0$ pick $k$-triangles $\Delta_{k} \subset E$ such that $\Delta_{k} \subset \Delta_{k+1}$ and $\bigcup_{k=0}^{\infty} \Delta_{k}=E$.

We will define inductively isometric embeddings $f_{k}: \Delta_{k}^{(1)} \rightarrow X^{(1)}$ such that $f_{k+1 \mid \Delta_{k}^{(1)}}=f_{k}$. The union $\bigcup_{k=0}^{\infty} f_{k}: E^{(1)} \rightarrow X^{(1)}$ will be the desired isometric embedding.

First, the hypothesis of the lemma guarantees that for arbitrarily large $n$ there exist isometric embeddings $D_{n}: \Delta_{n}^{(1)} \rightarrow X^{(1)}$. Since $\Gamma$ acts cocompactly on $X$, we can choose $\gamma_{n} \in \Gamma$ such that $\gamma_{n} \circ D_{n}\left(\Delta_{0}\right)$ belongs to a finite set of vertices in $X$. By passing to a subsequence and replacing $D_{n}$ with $\gamma_{n} \circ D_{n}$ we can ensure that $D_{n}\left(\Delta_{0}\right)$ does not depend on $n$. We then define $f_{0}: \Delta_{0} \rightarrow X^{(1)}$ by $f_{0}\left(\Delta_{0}\right)=D_{n}\left(\Delta_{0}\right)$.

Now suppose we have already defined an isometric embedding $f_{k}: \Delta_{k}^{(1)}$ $\rightarrow X^{(1)}$. Note that $\Delta_{k+1}^{(1)} \backslash \Delta_{k}^{(1)}$ is finite and $B_{1}\left(\operatorname{Im}\left(f_{k}\right)\right)$ is also finite (because $X$ is locally finite), so by passing to a subsequence we can ensure that $D_{n \mid \Delta_{k+1}^{(1)}}$ does not depend on $n$. We then define $f_{k+1}: \Delta_{k+1}^{(1)} \rightarrow X^{(1)}$ by $f_{k+1}=D_{n \mid \Delta_{k+1}^{(1)}}$. This ends the induction step.

4. Hyperbolicity. We are ready to prove the main theorem of the paper.

Proof of Theorem 1.2. One implication is easy. If $X^{(1)}$, the 1-skeleton of a systolic complex $X$, contains an isometrically embedded 1-skeleton of the 
triangulated Euclidean plane, then $X^{(1)}$ is not a hyperbolic metric space, so $\Gamma$ is not word-hyperbolic.

To prove the converse, suppose $\Gamma$ is not word-hyperbolic. Then, by a theorem of P. Papasoglu [6], bigons in $X^{(1)}$ are not thin, i.e. for every $n \in \mathbb{N}$ there exist vertices $V, Y \in X$ and two 1-skeleton geodesics $R, S$ joining $V, Y$ (denote their consecutive vertices by $V=R_{0}, R_{1}, \ldots, R_{m-1}, R_{m}=Y$; $\left.V=S_{0}, S_{1}, \ldots, S_{m-1}, S_{m}=Y\right)$ and there exists $t$ with $0<t<m$ such that $\left|R_{t} S_{t}\right|>n$. Set $k=\left|R_{t} S_{t}\right|>n$, choose a special 1-skeleton geodesic of length $k$ connecting $R_{t}, S_{t}$ and denote its consecutive vertices by $R_{t}=$ $P_{k}^{0}, P_{k}^{1}, \ldots, P_{k}^{k-1}, P_{k}^{k}=S_{t}$. Now construct inductively vertices $P_{i}^{j} \in X$, $0 \leq i, j \leq k, i+j \geq k$, in the following way. For $i=k$ the vertices are already given. Suppose we have already constructed vertices $P_{i}^{j}$ for all $i$ such that $p<i \leq k$, where $i, j$ are as above. Now we will define vertices $P_{i}^{j}$ for $i=p$. For each $j$ such that $k-p \leq j \leq k$ project the edge $P_{p+1}^{j-1} P_{p+1}^{j}$ onto the ball $B_{t-(k-p)}(V)$ and denote any vertex of this projection by $P_{p}^{j}$.

Now notice that for a fixed $l$ such that $0 \leq l \leq k$, the vertices $P_{i}^{j}$ with $i \geq k-l$ are all contained in the ball $B_{m-t+l}(Y)=B_{l}\left(B_{m-t}(Y)\right)$ and no other vertex $P_{i}^{j}$ belongs to this ball. This means that the $k$-triangle formed by the vertices $P_{i}^{j}$ satisfies all the assumptions of Lemma 3.3 with $Z=B_{m-t}(Y), D$ being the identity, and therefore the 1-skeleton of this $k$-triangle is isometrically embedded in $X^{(1)}$. Since $k>n$ can be chosen arbitrarily large, the hypothesis of Lemma 3.4 is satisfied and we obtain the 1-skeleton of the equilaterally triangulated Euclidean plane isometrically embedded in $X^{(1)}$.

REMARK 4.1. The existence of an isometric embedding of the 1-skeleton of an equilaterally triangulated Euclidean plane into $X^{(1)}$ does not imply we can embed the whole plane isometrically into $X$. For example consider $X$ equal to the equilaterally triangulated Euclidean plane with a cone over two adjacent triangles added.

\section{References}

[1] M. Bridson and A. Haefliger, Metric Spaces of Non-Positive Curvature, Grundlehren Math. Wiss. 319, Springer, 1999.

[2] T. Elsner, Flats and flat torus theorem in systolic spaces, in preparation.

[3] F. Haglund, Complexes simpliciaux hyperboliques de grande dimension, Prépublication Orsay 71, 2003.

[4] F. Haglund and J. Świątkowski, Separating quasi-convex subgroups in 7-systolic groups, submitted.

[5] T. Januszkiewicz and J. Świątkowski, Simplicial nonpositive curvature, Publ. Math. IHES, to appear. 
[6] P. Papasoglu, Strongly geodesically automatic groups are hyperbolic, Invent. Math. 121 (1995), 323-334.

[7] D. T. Wise, Sixtolic complexes and their fundamental groups, in preparation.

Faculty of Mathematics, Informatics and Mechanics

Warsaw University

Banacha 2

02-097 Warszawa, Poland

E-mail: pprzytyc@duch.mimuw.edu.pl

Received 20 June 2006 